\title{
Fractional-Order and Memristive Nonlinear Systems: Advances and Applications
}

\author{
Ahmed G. Radwan, ${ }^{1,2}$ Ahmad Taher Azar, ${ }^{2,3}$ Sundarapandian Vaidyanathan, \\ Jesus M. Munoz-Pacheco, ${ }^{5}$ and Adel Ouannas ${ }^{6}$ \\ ${ }^{1}$ Engineering Mathematics and Physics Department, Faculty of Engineering, Cairo University, Giza 12613, Egypt \\ ${ }^{2}$ NISC Research Center, Nile University, Giza 12588, Egypt \\ ${ }^{3}$ Faculty of Computers and Information, Benha University, Benha 13511, Egypt \\ ${ }^{4}$ Research and Development Centre, Vel Tech University, 400 Feet Outer Ring Road, Avadi, Chennai, Tamil Nadu 600062, India \\ ${ }^{5}$ Facultad de Ciencias de la Electrónica, Autonomous University of Puebla, Av. San Claudio y 18 Sur, Edif. FCE1, \\ 72570 Puebla, PUE, Mexico \\ ${ }^{6}$ Laboratory of Mathematics, Informatics and Systems, University of Larbi Tebessi, 12002 Tebessa, Algeria
}

Correspondence should be addressed to Ahmed G. Radwan; agradwan@ieee.org

Received 20 July 2017; Accepted 20 July 2017; Published 28 September 2017

Copyright (C) 2017 Ahmed G. Radwan et al. This is an open access article distributed under the Creative Commons Attribution License, which permits unrestricted use, distribution, and reproduction in any medium, provided the original work is properly cited.

Due to the significance of nonlinear systems and circuits in many applications such as physics, control, biophysics, and bioengineering, enormous research activities have been highlighted to model the practical and experimental insights of such phenomena. Chaotic systems are nonlinear dynamical systems which are sensitive to initial conditions, topologically mixing, and with dense periodic orbits [1-3]. The chaos phenomenon was first observed in weather models by Lorenz in 1963. This was followed by a discovery of a large number of chaotic systems in many different fields like computer sciences, mechanics, communication, economics and finance, biology, chemistry, medicine, and geology.

Recently, the developments of nonlinear circuits and memristors [4] have had a big impact on the way scientists and engineers apply analytical and computational techniques. In the last four years, many systems have been investigated using the innovative features of mem-elements in the analysis and designs of nonlinear circuits and systems, such as chaotic systems. Due to the difficulties of nonlinear circuit design, contributions towards new ideas, techniques, modeling, analysis, design, or fabrication will have a direct impact on future applications and the industry.

The chaotic dynamics of fractional-order systems began to attract a great deal of attention in recent years due to the ease of their electronic implementations. Due to the very high sensitivity of these chaotic systems which is required for many applications, there was a need to discuss the coupling of two or more dissipative chaotic systems which is known as synchronization. Chaotic synchronization has been applied in many different fields, such as biological, physical systems, structural engineering, and ecological models [1-4].

This special issue aims at presenting the latest developments, trends, research solutions, and applications of fractional-order and memristive chaotic systems with emphasis on real-world applications.

We received a total of twenty submissions, and after two rounds of rigorous review, seven papers were accepted.

In the paper "Fractional Order Memristor No Equilibrium Chaotic System with Its Adaptive Sliding Mode Synchronization and Genetically Optimized Fractional Order PID Synchronization," K. Rajagopal et al. introduce a new memristor based novel fractional order no equilibrium chaotic system (FOMNE) and investigate its adaptive sliding mode synchronization. Firstly the dynamic properties of the integer order memristor based novel no equilibrium system are analyzed. The fractional order memristor no equilibrium system is then derived from the integer order model. Lyapunov exponents and bifurcation with fractional order are investigated. An adaptive sliding mode control algorithm is derived to globally synchronize the identical 
fractional order memristor systems and genetically optimized fractional order PID controllers are designed and used to synchronize the FOMNE systems. Finally the fractional order memristor no equilibrium system is realized using FPGA.

In the paper "Generalized Fractional-Order DiscreteTime Integrator,” D. Mozyrska and P. Ostalczyk investigate a generalization of discrete-time integrator. The proposed linear discrete-time integrator is characterized by the variablefractional-order of integration/summation. Graphical illustrations are presented for an analysis of particular vector matrices. Numerical examples show the relations between the order functions and element responses.

In the paper "Dynamics, Circuit Design, and Synchronization of a New Chaotic System with Closed Curve Equilibrium," X. Wang et al. propose a new system with an infinite number of equilibrium points located on a closed curve. It is worth noting that the new system generates chaotic behavior as well as hidden attractors. Dynamics of the system with closed curve equilibrium have been investigated by using phase portraits, bifurcation diagram, maximal Lyapunov exponents, and Kaplan-York dimension. In addition, they introduce an electronic implementation to verify its feasibility. Antisynchronization ability of the new system with infinite equilibria is studied by applying an adaptive control. This study suggests that there exist other chaotic systems with uncountable equilibria in need of further investigation.

In the paper "Application of Topological Degree Method for Solutions of Coupled Systems of Multipoints Boundary Value Problems of Fractional Order Hybrid Differential Equations," M. Iqbal et al. have established the theory to a coupled systems of multipoints boundary value problems of fractional order hybrid differential equations with nonlinear perturbations of second type involving Caputo fractional derivative. For obtaining sufficient conditions on existence and uniqueness of positive solutions to the system under consideration, the authors have used the technique of topological degree theory. Finally, main results are illustrated by a concrete example.

In the paper "CMOS Realization of All-Positive Pinched Hysteresis Loops," B. J. Maundy et al. have proposed two novel nonlinear circuits that exhibit an all-positive pinched hysteresis loop. These circuits employ two NMOS transistors, one of which operates in its triode region, in addition to two first-order filter sections. The authors show the equivalency to a charge-controlled resistance (memristance) in a decremental state via detailed analysis. Simulation and experimental results verify the proposed theory.

In the paper "A Novel Chaotic System without Equilibrium: Dynamics, Synchronization, and Circuit Realization," A. T. Azar et al. have proposed a novel unusual chaotic system without equilibrium. The authors discover dynamical properties as well as the synchronization of the new system. Furthermore, a physical realization of the system without equilibrium is also implemented to illustrate its feasibility.

In the paper "Understanding the Resistive Switching Phenomena of Stacked $\mathrm{Al} / \mathrm{Al}_{2} \mathrm{O}_{3} / \mathrm{Al}$ Thin Films from the Dynamics of Conductive Filaments," J. Molina and L. Hernandez-Martinez present the resistive switching characteristics of
Metal-Insulator-Metal (MIM) devices based on amorphous$\mathrm{Al}_{2} \mathrm{O}_{3}$ and deposited by Atomic-Layer Deposition (ALD). A maximum processing temperature for this memory device is $300^{\circ} \mathrm{C}$, making it ideal for Back-End-Of-Line (BEOL) processing. Even though some variations in the forming, set, and reset voltages $\left(V_{\mathrm{FORM}}, V_{\mathrm{SET}}\right.$, and $\left.V_{\mathrm{RES}}\right)$ are obtained for many of the measured MIM devices (mainly due to roughness variations of the MIM interfaces as observed after atomic-force microscopy analysis), the memristor effect has been obtained after cyclic $I-V$ measurements. These resistive transitions in the metal oxide occur for both bipolar and unipolar conditions while the $I_{\mathrm{OFF}} / I_{\mathrm{ON}}$ ratio is around 4-6 orders of magnitude and is formed at gate voltages of $\mathrm{Vg}<4 \mathrm{~V}$. In unipolar mode, a gradual reduction in VSET is observed and is related to a combined (a) incomplete dissolution of conductive filaments (made of oxygen vacancies and metal ions) that leaves some residuals and (b) thickening of chemically reduced $\mathrm{Al}_{2} \mathrm{O}_{3}$ during localized Joule heating. In conclusion, low-thermal budget MIM based memories using $\mathrm{Al} / \mathrm{Al}_{2} \mathrm{O}_{3} / \mathrm{Al}$ structures have been fabricated and the memristor effect has been observed for the bipolar and unipolar switching modes, which are dependent on the amount of electrons tunneling through the device.

\section{Acknowledgments}

The guest editorial team would like to thank authors of all the papers submitted to this special issue. The editors also wish to thank the anonymous reviewers, some of whom helped with multiple review assignments. Finally, we would like to thank the journal's Editorial Board for being very encouraging and accommodative regarding this special issue. We hope that you will enjoy reading this special issue devoted to this exciting and fast-evolving field as much as we have done.

\section{Ahmed G. Radwan \\ Ahmad Taher Azar \\ Sundarapandian Vaidyanathan Jesus M. Munoz-Pacheco \\ Adel Ouannas}

\section{References}

[1] A. T. Azar and S. Vaidyanathan, Chaos Modeling and Control Systems Design, vol. 581 of Studies in Computational Intelligence, Springer, Berlin, Germany, 2015.

[2] A. T. Azar and S. Vaidyanathan, Advances in Chaos Theory and Intelligent Control, vol. 337 of Studies in Fuzziness and Soft Computing, Springer, Berlin, Germany, 2016.

[3] A. T. Azar, S. Vaidyanathan, and A. Ouannas, Fractional Order Control and Synchronization of Chaotic Systems, vol. 688 of Studies in Computational Intelligence, Springer, Berlin, Germany, 2017.

[4] A. G. Radwan and M. E. Fouda, On The Mathematical Modeling of Memristor, Memcapacitor, and Meminductor, vol. 26 of Studies in Systems, Decision and Control, Springer, 2015. 


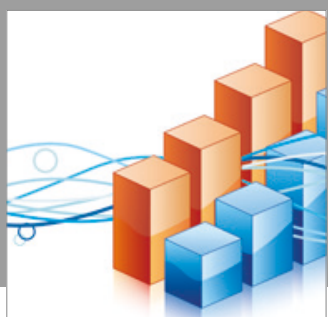

Advances in

Operations Research

vatersals

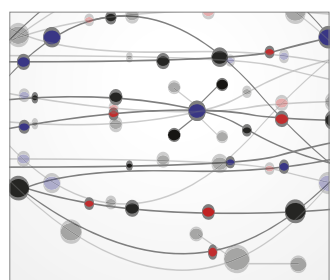

\section{The Scientific} World Journal
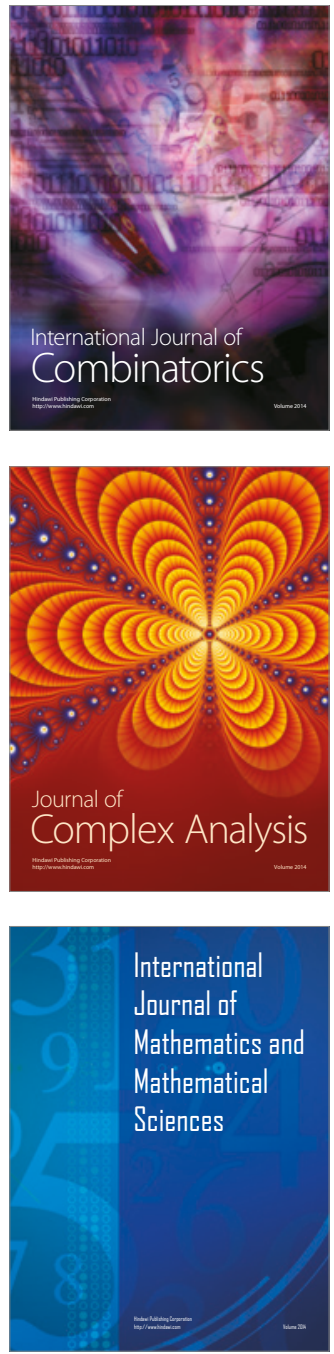
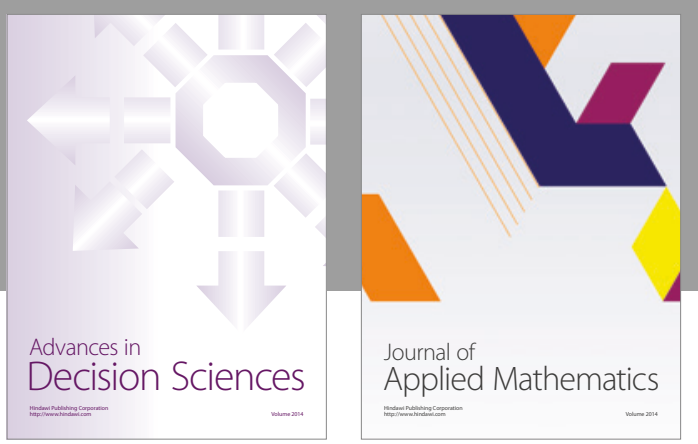

Algebra

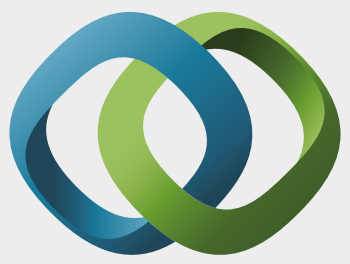

\section{Hindawi}

Submit your manuscripts at

https://www.hindawi.com
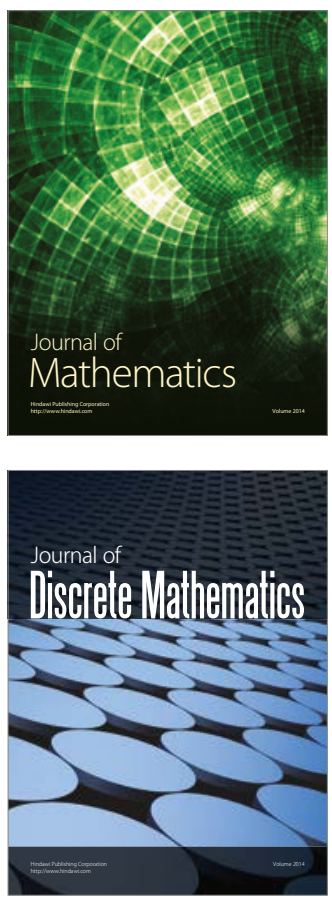

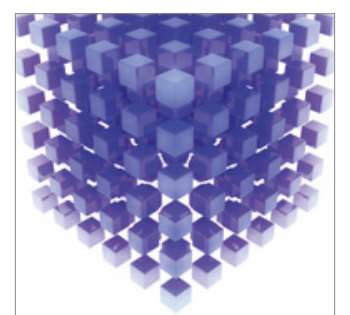

Mathematical Problems in Engineering
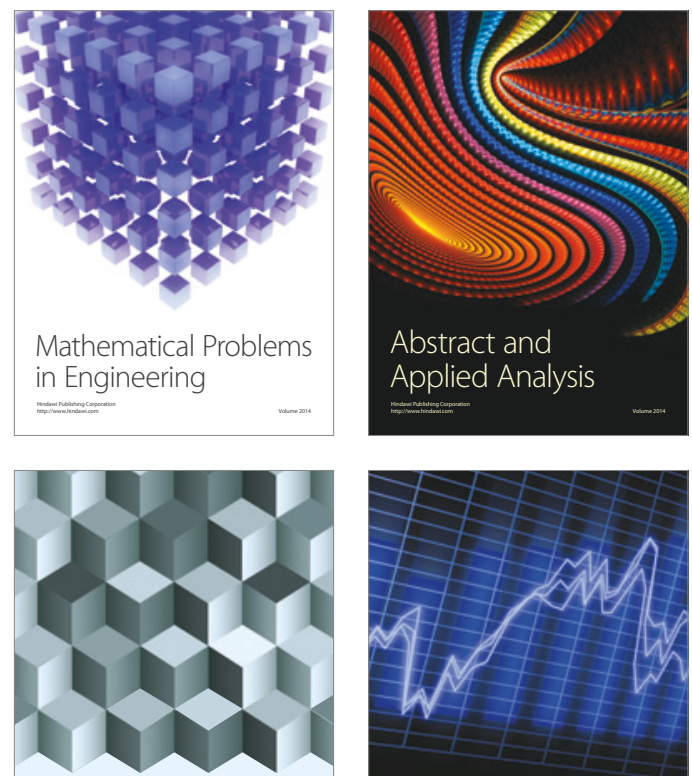

Journal of

Function Spaces

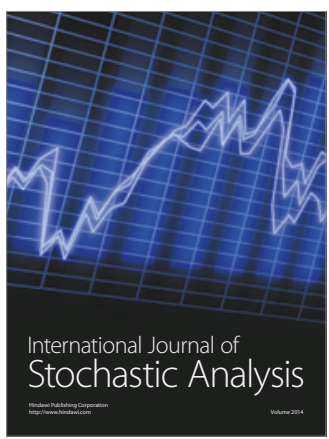

Probability and Statistics
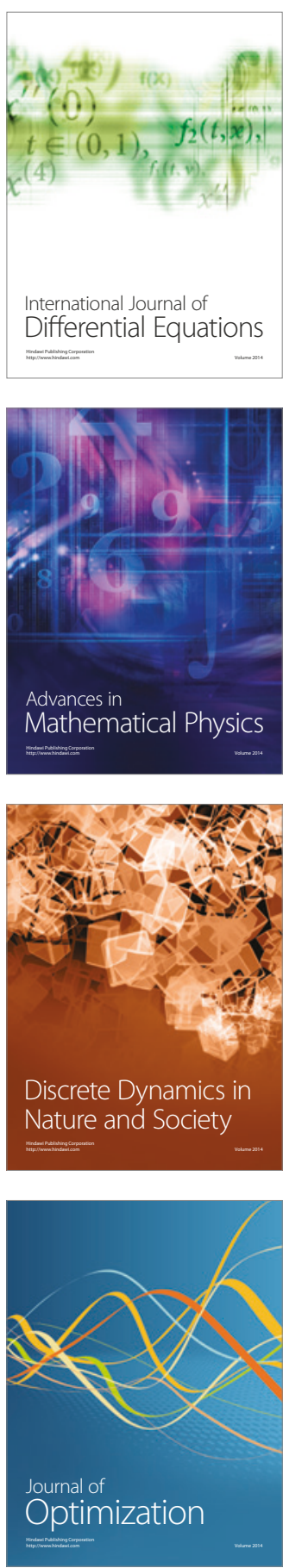